\title{
3 Die Evolutionstheorie als Rahmen und Hintergrund der Sprachursprungsforschung
}

\author{
Nothing in Biology Makes Sense Except in the Light of Evolution[.]
}

Dobzhansky (1973: 125)

\subsection{Die Evolutionstheorie und ihr Platz in der modernen Sprachursprungsforschung}

Sicherlich nimmt der Begriff Evolution prinzipiell auch innerhalb des Lexikons eines wissenschaftlichen Laien eine durchaus bedeutsame Position ein. Eine Verwendung des Begriffs in einer derartigen - d.h. wissenschaftlich unverbindlichen und daher semantisch vergleichsweise vagen - Lesart im Sinne einer ,langsam fortschreitenden Entwicklung ermöglicht einen vielseitigen Gebrauch, mitunter im übertragenen Sinn, innerhalb der alltäglichen Sprachverwendung. Von der Evolution der Politik über die Evolution der Popmusik bis zu derjenigen des Fußballs finden sich naive Bezeichnungen bzw. Beschreibungen in dem Versuch, die Aspekte der Chronologie und der Wandlung hinsichtlich der historischen Entwicklung einer konkreten Sache bzw. Thematik in Verbindung zu bringen, wiederkehrend im populären Gebrauch.

Obwohl keinesfalls deckungsgleich, so ist freilich auch in dieser recht freizügigen Nutzung des Begriffs der Evolution eine wenigstens rudimentäre Verbindung mit dem wissenschaftlich bedeutsameren Begriff der biologischen Evolution durchaus gegeben. Dennoch impliziert eine derartige Verbindung respektive ein derartiges Verständnis nicht zwingend eine für das vorliegende Unterfangen angemessene Kenntnis über zentrale Prinzipien und Vorgänge der Evolutionstheorie im wissenschaftlich bedeutsameren, biologischen Sinn. Mithin zeigen sich viele der zentralen Prinzipien der modernen Evolutionstheorie gegenüber einem Verständnis durch einschlägig nicht oder wenig vorgebildete Laien als ebenso opak wie beispielsweise jene Prinzipien, die innerhalb der theoretischen Physik der Gravitationstheorie zugrunde liegen, obgleich Gravitation ebenfalls eine prominente Begrifflichkeit im Lexikon sowie innerhalb der Alltagserfahrung eines jeden wissenschaftlichen Laien darstellt.

Im Kontext dieser Feststellungen gilt weiterhin zu betonen, dass für eine Diskussion zum Ursprung menschlicher Sprachfähigkeit, wenn sie denn ohne größere Missverständnisse geführt werden soll, durchaus erforderlich ist, in einen Verständniskontext eingebettet zu sein, der unter anderem die moderne Evolutionstheorie umfasst. Dies gilt insbesondere für die am Forschungsge- 
schehen beteiligten bzw. die zum Forschungsgeschehen beitragenden Diskutanten, aber gewiss ferner für ein jedes Individuum, welches gedenkt, die einschlägige Forschung zu rezipieren, und welches im Rahmen dieses Vorhabens sucht, defizitäre Spekulation und defiziente Interpretationen aufgrund fahrlässiger Ignoranz gegenüber der modernen Evolutionstheorie zu vermeiden bzw. als solche zu erkennen.

Hierbei handelt es sich freilich weder um eine willkürliche noch um eine innerhalb der Forschung außergewöhnliche Feststellung. Stattdessen lässt sich diese Auffassung aus dem weithin gebräuchlichen Vorgehen, die Festigung menschlicher Sprachfähigkeit innerhalb der menschlichen evolutionären Linie durch primär evolutionsbiologische Prozesse zu erklären, ableiten - ein Vorgehen, dem eine lange Tradition innewohnt (vgl. Darwin 1871/1889 und 1872), dabei jedoch in seiner modernen Form auf eine einflussreiche Veröffentlichung von Pinker \& Bloom (1990) zurückgeht und innerhalb der Sprachursprungsforschung gemeinhin implizit oder explizit vertreten wird. Insbesondere innerhalb der relevanten evolutionsbiologischen Literatur zeigt sich diese Ansicht quasi als eine Selbstverständlichkeit (vgl. bspw. Maynard Smith \& Szathmáry 1996, insbesondere 296-297, für ein akademisch in die Evolutionsbiologie einführendes Werk, welches sich dabei auch knapp auf die Evolution von Sprachfähigkeit bezieht).

Dieses Heranziehen der biologischen Evolution als Erklärungshintergrund zur menschlichen Sprachbefähigung basiert auf der großen Plausibilität des folgenden Gedankengangs: Die biologischen Grundlagen zur menschlichen Sprachfähigkeit gestalten sich umfangreich sowie mannigfaltig und stehen in komplexen Beziehungen zu weiteren biologischen Vorgängen bzw. funktionalen Komplexen. Demgemäß liegt die naheliegende Feststellung darin, dass eine Umgestaltung und Erweiterung der relevanten Biologie hin zur modernen Sprachfähigkeit großflächig sowie vielseitig vonstattengegangen sein muss ein Prozess, wie er in besonderem Maße im Licht der modernen Evolutionstheorie plausibel und zugleich fruchtbar, d.h. in Übereinstimmung mit den Erkenntnissen der beteiligten Wissenschaften sowie mit nennenswerter Aussageund Erklärungskraft, beschrieben werden kann.

Zusätzlich klärend und zugunsten der Vollständigkeit sei anzumerken, dass diese generelle Position innerhalb der einschlägigen Literatur auf zweierlei Weise kontrastiert wird: Einerseits widersprechen Autoren, die von einer primär oder vollständig kulturellen Basis moderner Sprachfähigkeit sowie Sprachen ausgehen und in diesem Sinne einen engen Zusammenhang mit Prozessen der biologischen Evolution verneinen (vgl. für eine Position der jüngeren Forschung: Christiansen \& Chater 2008, Chater, Reali \& Christiansen 2009 sowie 
Chater \& Christiansen 2010), und andererseits geschieht eine Zurückweisung biologischer - bzw. genauer adaptiver, selektiver - Evolution hinsichtlich menschlicher Sprachfähigkeit auch durch Autoren, welche die Möglichkeit protosprachlicher Zwischenstufen innerhalb der Entwicklung von nichtmenschlicher Kommunikation zu menschlicher Sprachfähigkeit strikt ausschließen (vgl. bspw. Bickerton 1990 trotz seiner damals wegweisenden Einführung des Konzepts der Protosprache in die einschlägige Diskussion und jüngst, als zwischenzeitliche Kulmination einer längeren Tradition, Berwick \& Chomsky 2016). In diesem Sinne sei die Platzierung einer modernen und mitunter durchaus biologisch begründeten Sprachfähigkeit eine vergleichsweise plötzliche Angelegenheit gewesen, womit eine Interpretation im Sinne evolutionsbiologischer Selektion bzw. des Prozesses der Adaptation, d.h. einer sukzessiven und differenzierten Entwicklung einschließlich den erwähnten protosprachlichen Zwischenstufen, auszuschließen sei. Diese allzu knappen und daher analytisch unscharfen Skizzen sollen im gegebenen Kontext nicht weiter ausgeführt oder diskutiert werden, da sie im späteren Verlauf der vorliegenden Arbeit wenigstens teilweise detailliert zur Diskussion stehen werden.

Stattdessen ist weiterhin klärend anzumerken, dass mit den mahnenden Worten eines notwendig erforderlichen Kenntnishintergrunds zur Evolutionsbiologie freilich kein zur Forschung befähigendes, technisches Expertenwissen gemeint sein soll. Eine derart tiefgehende Expertise kann von einem einzelnen Individuum ohnehin nicht für alle zur Forschungsfrage des Ursprungs des menschlichen Sprachvermögens beitragenden Disziplinen erreicht werden. Dennoch ist ein fundiertes Verständnis zu grundlegenden Aspekten der Theorie in ihrer modernen Form von nennenswerter Bedeutsamkeit für ein verortendes Gesamtverständnis. Das vorliegende Kapitel stellt den Versuch dar, ebenjene Aspekte in knapper und dennoch präziser Form zu skizzieren, um einen adäquaten Verständnishintergrund bzw. -kontext zur Sprachursprungsforschung zu schaffen. Da die nachfolgenden Ausführungen im Rahmen des gegebenen Vorhabens in Umfang als stark eingegrenzt $\mathrm{zu}$ sehen sind, sei mit Dawkins (1986/2006), Gould (2002), Carroll (2006), Prothero (2007) und Sterelny (2007) beispielhaft auf umfassende, populärwissenschaftliche Einführungen zur modernen Evolutionstheorie sowie $\mathrm{zu}$ noch immer diskutierten Fragestellungen innerhalb der einschlägigen Forschung verwiesen. Für zwei akademisch orientierte deutschsprachige Einführungen siehe Maynard Smith \& Szathmáry (1996) sowie Kutschera (2001/2015). 


\subsection{Die gedanklichen Fundamente der modernen Evolutionstheorie}

Zunächst seien in diesem Programm die Begrifflichkeiten einer bzw. der Evolutionstheorie $\mathrm{zu}$ definieren und Vorreiter der modernen Theorie angemessen zu verorten. Dies ist dahingehend von Wert, als dass das öffentliche Verständnis die moderne Evolutionstheorie oftmals mit dem vulgarisierenden Begriff eines „Darwinismus“ gleichsetzt - d.h. mit einem statischen und dogmatischen Weltbild. Dagegen steht die historische Realität, nach welcher der Weg zur modernen, synthetischen Evolutionstheorie sowohl vor als auch nach Darwin von einer Vielzahl kleinerer und größerer Erkenntnisschritte begleitet wurde. Mithin zeugt ein kleiner historischer Abriss, wie er nun folgen soll, von der Leistungsfähigkeit des wissenschaftlichen Fortschritts hin zur aktuellen Lehrmeinung und fördert zugleich ein inhaltliches Verständnis der modernen Evolutionstheorie - trotz der freilich gezielt exemplarischen Natur und der großen Knappheit jener historischen Skizze sowie der darin enthaltenen konzeptuellen Aufbereitung. In jedem Fall vermag die historische Tiefe evolutionsbiologischnaturphilosophischer Ideen es, einen aufklärenden Einfluss auf ein einschlägiges Gesamtverständnis zu haben.

So gehen naturphilosophische Positionen, die der bzw. den neuzeitlichen Evolutionstheorie(n) hinsichtlich einzelner Aspekte vorgreifen, innerhalb der westlichen Denktradition bis in die griechische Antike zurück, ohne dabei jedoch den in der jüngeren Wissenschaftsgeschichte gängigen Ansprüchen gegenüber einer wissenschaftlichen Theorie zu genügen. Dies ist derart festzuhalten, da eine wissenschaftliche Theorie - gegenüber der alltagssprachlichen Verwendung des Begriffs der Theorie als eine mehr oder weniger gut fundierte spekulative Feststellung - ein hohes Maß an interner Systematizität beinhaltet und einen starken empirischen Bezug erfordert, durch den sie fundiert werden muss, bevor sie in Betracht gezogen werden kann (vgl. für die Definition einer wissenschaftlichen Theorie die Ausführungen der amerikanischen National Academy of Sciences (1999) oder durch The Stanford Encyclopedia of Philosophy (Winther 2015) - Literaturangaben im Literaturverzeichnis; vgl. für eine deutschsprachige Einführung zur unterliegenden Wissenschaftstheorie bspw. Balzer 1997). Vorneuzeitliche Ideenkonstrukte beinhalten zwar oftmals eine große interne Systematizität - auch wenn dies hinsichtlich vieler Philosophen des antiken Griechenlands, darunter insbesondere der vorsokratischen Philosophen, durchaus angezweifelt werden kann - , vermissen jedoch beinahe ausnahmslos den konsequenten Bezug zur Empirie. 
Obwohl daher nicht als „Theorie“ zu bezeichnen, so beinhalten einige naturphilosophische Positionen der griechischen Antike dennoch eine Reihe von Aspekten und Perspektiven, die in Gegenüberstellung zu Weltanschauungen, welche eine absolute sowie unveränderliche Weltstruktur postulieren und welche unzweifelhaft während des Großteils der zivilisierten Menschheitsgeschichte - vom Altertum bis weit in das 19. Jahrhundert hinein - im alltäglichen, religiösen sowie (natur)philosophischen Diskurs vorherrschend waren, sicherlich als richtungsweisend anzusehen sind. Dazu zu zählen ist die Veränderlichkeit der natürlichen Ordnung in einem eher generellen Sinn ebenso wie die prinzipielle Veränderlichkeit von Organismen sowie eine potenzielle gemeinsame Abstammung von Lebensformen aus simpleren Lebensformen und die Zunahme von Komplexität über Zeit bzw. im Verlauf von aufeinander folgenden Generationen. Aufgrund der Vorreiterrolle, die hinsichtlich dieser Naturphilosophen gegenüber späteren Gedankenkonstrukten festzustellen ist, sollen ausgewählte Vorsokratiker hiermit beispielhaft erwähnt und relevante Teile ihrer Positionen in knappster Form skizziert werden.

Als ein erster historischer Vorreiter ist hier Thales von Milet zu nennen, welcher entgegen der seinerzeit vorherrschenden Weltanschauung über einen immateriellen, religiös begründeten Ursprung der Welt und der vorliegenden Weltordnung das Wasser als den materiellen Urgrund aller Dinge ansah (vgl. in Ermangelung von Primärquellen als historische Sekundärquelle Aristoteles 983b bzw. zusätzlich Mansfeld 1983/1999, insb. 39-40, sowie Mansfeld 1985 für moderne Erläuterungen zu Thales' historischer Bedeutung). In Folge konstituieren sich alle weiteren Phänomene oder Substanzen durch eine Wandlung bzw. Umformung aus diesem materiellen Ausgangspunkt. Obwohl sicherlich aus heutiger Sicht naiv und fehlgeleitet, so ist anzuerkennen, dass Thales auf diese Weise erstens die natürlichen Phänomene entmythologisierte und zweitens die natürliche Weltordnung als inhärent, d.h. ohne Einflüsse durch göttliche Intervention, veränderlich charakterisierte. In anderen Worten: Mit Thales' Feststellungen wurde eine wegweisende und einflussreiche Denktradition geschaffen, welche nicht nur den Großteil der ihm nachfolgenden vorsokratischen Naturphilosophen maßgeblich lenkte, sondern die Grundlagen für philosophische Diskussionen legte, welche schlussendlich in der Evolutionstheorie Darwins kulminierten. Auch Mansfeld (1983/1999: 40) stellt Vergleichbares fest: „Thales darf also als der entscheidende Wegbereiter des Begriffs des natürlichen Prozesses [...] betrachtet werden.“

Obwohl dessen Schüler Anaximander zwar im Kontrast zu Thales von Milet wiederum in die ältere Tradition, einen immateriellen Urstoff $\mathrm{zu}$ postulieren, verfiel, so schlug er dennoch das Feuchte als den Ursprung simpler Lebensfor- 
men vor - Lebensformen, die sich durch Metamorphosen wandeln konnten und sukzessive die moderne tierische Vielfalt hervorbrachten (Mansfeld 1983/1999: 62-65 und Gregory 2016: 29-50). Die Bedeutung des eben Gesagten soll nochmals betont werden: Die Ideen des Thales weiterentwickelnd fixierte Anaximander erstmals eine Position, welche die schrittweise Veränderlichkeit der Tierwelt explizit festhielt. Einer der späteren Vorsokratiker, Empedokles, schlug des Weiteren vor, dass in vor-historischer Zeit zunächst Pflanzen und erst später Tiere existierten. Auch nahm er an, dass zunächst simple Lebensformen bzw. teilweise Formen entstanden und sich durch Kombination fortzupflanzen gedachten. Nur diejenigen Formen, die auch wirklich zusammenpassten, konnten überleben und brachten so die zu seiner Zeit bestehende natürliche Ordnung zustande (Mansfeld 1986/1999: 60-61 und 65 sowie Campbells Eintrag „Empedocles“ in der Internet Encyclopedia of Philosophy; für weiterführende Angaben siehe das Literaturverzeichnis).

Obwohl jene Versuche, die Weltordnung mit einer inhärenten Veränderlichkeit - mitunter auch bezogen auf nicht-menschliche Tiere sowie menschliche bzw. vormenschliche Lebensformen - zu erklären, so sind sie dennoch als naiv, wenig systematisch und empirisch nicht bis kaum belegt einzuschätzen. Wie bereits angemerkt wurde, sind gängige Definitionen zu wissenschaftlichen Theorien als zu streng anzusehen, als dass jene oder auch spätere naturphilosophische Gedankenkonstrukte der Antike, des Mittelalters oder der frühen Neuzeit hinzuzuzählen wären. Mitunter aufgrund dieser spekulativen, philosophischen Natur konnten sich die erwähnten sowie weitere Positionen historisch nicht großflächig durchsetzen. Hinzu kam als weiterhin erschwerender, ja für näherungsweise zwei Jahrtausende als geradezu unüberwindlich einzuschätzender Faktor, dass sich die christliche Kirche in der Spätantike nicht nur als religiös, sondern ebenso als philosophisch und akademisch dominierender Erklärungshintergrund aller nennenswerter Diskussion zum Ursprung des Weltganzen und zur inhärenten Ordnung der Natur etablierte - innerhalb der Kosmologie ebenso wie hinsichtlich der Geologie, der Biologie und sonstig relevanten Perspektiven. Die damit verbundene generelle Weltanschauung einer gottgeschaffenen und damit vollständig intendierten sowie unveränderlichen Natur führte zu einer kulturellen Tradition, in der mit jenen Vorsokratikern verwandte Denkansätze im Keim erstickt wurden und in der jenseits der privaten Kommunikation einiger weniger Gelehrter derlei Gedankenkonstrukte keine populäre Verbreitung finden konnten. 


\subsection{Die neuzeitliche Evolutionsbiologie vor Darwin}

Mit diesen Worten sei hinsichtlich der Skizze zu den ersten zaghaften naturphilosophischen Positionen ein Abschluss gefunden, womit im Folgenden zu einer engeren Auslegung des Begriffs Evolution bzw. Evolutionstheorie fortzuschreiten ist. In populären Beschreibungen der einschlägigen geschichtlichen Hintergründe wird als der erste Vertreter der Moderne, welcher eine prominente, systematische und empirisch fundierte Evolutionstheorie vorschlug, gemeinhin Lamarck (1809) genannt. Hierzu ist zunächst anzumerken, dass Lamarcks Ausführungen, wenn verglichen mit denen von Darwin und späteren Autoren zur modernen Evolutionstheorie, in noch immer lediglich geringem Maße einen empirischen Bezug hatten und auch hinsichtlich ihrer internen Systematizität oftmals überschätzt wird - wie nicht nur aus moderner Sicht, sondern bereits zeitgemäß, bspw. durch Lyell, ${ }^{4}$ festgestellt wurde. Trotz dieses Umstands stellt Lamarck sicherlich die historisch einflussreichste und populärste Alternative zu Darwins Evolutionstheorie dar und soll schon allein aus diesem Grund knapp skizziert werden.

Zugleich sollte hierzu klärend angemerkt werden, dass Lamarcks besondere Stellung maßgebend davon herrührt, dass im Gegenentwurf zu Darwin noch im 19. Jahrhundert eine Bewegung entstand, welche sich einem Neo-Lamarckismus zuschrieb und welche dabei lediglich eine teilweise Deckungsgleichheit mit Lamarcks tatsächlicher Position aufwies. Dieser Neo-Lamarckismus wirkte jahrzehntelang mit großem Einfluss innerhalb der Evolutionsbiologie des späten 19. sowie frühen 20. Jahrhunderts und wird in der populären Kultur sowie in vielen Schulbüchern ungerechtfertigter Weise als praktisch synonym mit dem Namen Lamarck genannt. Hierauf wird im Folgenden freilich Rücksicht genommen, jedoch soll zugleich auf eine weitergehende Diskussion verzichtet werden. Erwähnt seien als weiterführende Quellen für eine Diskussion zu der Problematik einer populären Verfälschung von Lamarcks Evolutionstheorie Ghiselin (1994), Gould (2002: 172) und Mayr (1972: 86). Im gleichen Zuge gilt klärend anzumerken, dass die generelle Denktradition einer veränderlichen Weltordnung bzw. Tierwelt wie zuvor dargestellt bis in die Antike zurückzuverfolgen ist und unter Philosophen sowie Biologen des 18. und 19. Jahrhunderts gemeinhin bekannt war. Lamarcks Beitrag war damit nicht der Ursprung dieses Gedankenguts, sondern primär die gesammelte und stringente Aufbereitung einschlägiger

4 Lyell beschäftigte sich besonders in Band II der Erstauflage seiner Principles of geology (in drei Bänden: 1830, 1832 und 1833) mit Lamarck (Innerhalb seiner Neuveröffentlichung in vier Bänden ist diese Kritik konkret wie folgt zu finden: 1835, Band II: 326-366). 
Tendenzen seiner Zeit verflochten mit teilweise in evolutionsbiologischer Hinsicht durchaus progressiven Überlegungen.

Der historische Hintergrund $\mathrm{zu}$ Lamarck gestaltete sich dabei wie folgt: Im Licht vermehrter Fossilfunde im Verlauf der frühen Neuzeit sowie im Kontext erster systematischer Taxonomien wie derjenigen durch von Linné (1735/1766) waren sowohl die Existenz vergangener Ökosysteme als auch die Verwandtschaft vieler taxonomischer Gruppen kaum mehr zu verneinen. Anders als beispielsweise durch Cuvier (1825) innerhalb dessen Kataklysmen- bzw. Katastrophentheorie, welche die prinzipielle Unveränderlichkeit existenter Ökosysteme $\mathrm{zu}$ erhalten suchte, vorgeschlagen, postulierte Lamarck die gradualistische Veränderlichkeit von Arten und verband diesen zentralen Aspekt mit einer zu seiner Zeit angemessen systematischen Theorie. Seine Vorreiterrolle ist insbesondere auf zwei Wegen zu belegen: Erstens war Lamarck sicherlich einer der zentralen Einflüsse auf Darwins spätere Evolutionstheorie - sowohl hinsichtlich übereinstimmender als auch hinsichtlich in Kontrast stehender Aspekte der beiden Theorien (vgl. Mayr 1972: 90). Zweitens waren Lamarcks Ausführungen wie bereits angemerkt hinreichend stringent, um in Form eines NeoLamarckismus von einem nennenswerten Anteil der Forschungsgemeinschaft als scheinbar plausible Alternative zum Neo-Darwinismus bis weit in das 20 . Jahrhundert hinein diskutiert zu werden. Erst im Verlauf der 1920er und 1930er Jahre wandelte sich dieser Neo-Lamarckismus vollständig zu einer Randerscheinung. ${ }^{5}$

Lamarcks Evolutionstheorie war dabei geprägt von einer gewissen inneren Unausgewogenheit bzw. Inkonsequenz. Auf der einen Seite waren seine systematischen Feststellungen zur prinzipiellen Veränderlichkeit von Organismen im Kontext von dynamischen ökologischen und geologischen Systemen sowie zur gradualistischen Veränderung über aufeinander folgende Generationen sicherlich fortschrittliche Gedanken - jedenfalls, wenn verglichen mit den zeitgemäßen Lehrmeinungen (Ghilarov 1998, Mayr 1972). Auf der anderen Seite begründete sich Lamarcks Position auf einem geradezu mystischen „Vervollkommnungstrieb“, d.h. einen Individuen inhärent gegebenen „Richtungssinn“, dem die existierenden Arten nachstreben. Darüber hinaus zeigte sich Lamarck

5 Diese Feststellung bezieht sich auf den sich innerhalb dieses groben Zeitraums einstellenden wissenschaftlichen Konsens im Zuge des zunehmend erstarkenden Feldes der Genetik und erfährt keine Minderung hinsichtlich ihrer Validität durch einen Verweis auf die vorübergehende und rein ideologisch, d.h. nicht wissenschaftlich, begründete Wiederbelebung des NeoLamarckismus in der Sowjetunion der 1940er und 1950er Jahre. Vgl. zur geschichtlichen Nachverfolgung der Wiederbelebung jenes Gedankenguts sowie zur Biographie der hierfür primär verantwortlichen Persönlichkeit beispielsweise Medwedjew (1969/1982). 
hinsichtlich der gemeinsamen Abstammung von Arten gegenüber den Tendenzen seiner Zeit kompromissbereit: Nicht alles Leben sei miteinander verwandt, sondern begann im Kontext einer hinsichtlich ihrer Systematik bereits ausdifferenzierten Urzeugung. Aus diesen prototypischen Lebewesen entwickelten sich einzelne Arten fortschreitend in Richtung zunehmender Komplexität entlang der Linie ihres innerlich gegebenen Richtungssinns bzw. Vervollkommnungstriebs. Freilich handelt es sich hierbei um archaische und fehlgeleitete, aus heutiger Sicht pseudowissenschaftliche Überlegungen. Zuletzt zeigte sich schließlich auch der dem Lamarckismus unterliegende Mechanismus der Modifikation von Organen durch die Gewohnheiten von Tieren sowie die Vererbung dieser erworbenen Modifikationen, also die populär bekannte lamarckische Idee der „Veränderung durch Gebrauch bzw. Nichtgebrauch“, als unzutreffend. ${ }^{6}$ Insgesamt ist daher der Lamarckismus - nebst dem späteren NeoLamarckismus - als ein gescheitertes Programm zu sehen (vgl. Mayr 1972 für eine kritische und dabei durchaus auch anerkennende Rückschau auf Lamarck einschließlich der soeben vorgebrachten Punkte).

\subsection{Darwins Evolutionstheorie und deren Limitationen}

Schlussendlich ist der Begriff der Evolutionstheorie nochmals in einem engeren Sinn fassbar, sodass er gezielt auf die Evolutionstheorie nach Darwin anzuwenden ist. Obgleich in Darwins Ausführungen hinsichtlich einiger Aspekte Übereinstimmung mit Lamarck bestand, so waren es neben seinen primär empirischen Methoden - wie vormals erwähnt war Lamarcks Vorgehensweise in wesentlich größerem Maße philosophisch bzw. naturphilosophisch geprägt als Darwins streng empirisch fundiertes Vorgehen - insbesondere diejenigen Feststellungen, mit denen sich Darwin von Lamarck abhob, welche sich ultimativ als wegweisend und weithin zutreffend erwiesen und welche Darwins Status als Begründer der modernen Evolutionstheorie fundierten. Dazu zählen beispielsweise die Betonung einer vollends gemeinsamen Abstammung aller Lebewesen, der Ausschluss des bei Lamarck noch postulierten mystischen Vervollkommnungstriebs von Lebensformen und der Mechanismus der natürlichen sowie

6 Erkenntnisse der jüngeren Forschungsgeschichte, welche unter dem fachterminologischen Mantel der Epigenetik zusammengefasst werden und welche die Relevanz vererbter Eigenschaften für die einschlägige Forschung innerhalb der zweiten Hälfte des 20. Jahrhunderts in begrenztem Umfang wiederherstellte, können im gegebenen Kontext nicht berücksichtigt werden. Die im gegebenen Kontext angebrachte Erwähnung jenes Forschungsbereichs sei hiermit geschehen. 
sexuellen Selektion im Kontext von Variation und „Überproduktion“ von Individuen innerhalb einer Population. Dennoch ist Darwin nicht als der Endpunkt, für den er innerhalb der populären Meinung oftmals gehalten wird, zu sehen, sondern vielmehr als ein weiteres Trittbrett in einer Reihe von einflussreichen Denkern hin zur modernen synthetischen Evolutionstheorie, welche Darwins historische Position hinsichtlich einiger Aspekte obsolet machte.

So umfassen beispielsweise Darwins vorgeschlagene Formen der Selektion nicht das gesamte Spektrum selektiver Einflüsse und Darwins vorgeschlagener Vererbungsmechanismus der Pangenesis ${ }^{7}$ zeigte verfälschende Reste lamarckischen evolutionären Denkens (Mayr 1972: 78). Nicht zuletzt fehlten Darwin vollumfänglich die Erkenntnisse aus der modernen Genetik mitsamt aller sich daraus erschließenden Beschreibungsebenen der Evolutionsbiologie. In diesem Sinne erfährt die Evolutionstheorie bis heute irregulär und verschiedentlich umfangreich Modifikationen gemäß der beständig fortschreitenden Forschungslage und ist nicht durch eine dogmatische Fixierung gebunden (für ein Beispiel zu einer intensiv geführten Diskussion der jüngeren Forschungsgeschichte zwischen Positionen, die jeweils durch Dawkins und Gould angeführt wurden, vgl. Sterelny 2007). Diesem wachsenden Verständnis innerhalb der beteiligten Einzelwissenschaften steht eine eher stagnierende und simplifizierte Vorstellung des Laien gegenüber. Nachfolgend soll daher die zentrale Dynamik, mit der sich der evolutionäre Wandel vollzieht, skizziert werden. Dabei finden auch einige bedeutsame Prinzipien der modernen Evolutionstheorie in einer nicht sonderlich technischen Form Erwähnung. In dieser Weise sollen Missverständnisse gegenüber dem evolutionären Vorgang sowie der modernen Evolutionstheorie aufgeklärt werden.

Zugleich soll nochmals explizit betont werden, dass sich die nachfolgenden Erläuterungen stark einschränken müssen. Das evolutionsbiologische Programm soll knapp skizziert werden, um ein treffendes, jedoch zugleich noch immer oberflächliches Verständnis zu vermitteln. Zugrunde liegende Prozesse, die der Genetik zugehören, sowie hintergründige mathematische Modelle und Interpretationen verbleiben zugunsten der Übersichtlichkeit weitgehend unerwähnt. Für eine umfassende deutschsprachige Einführung in die Genetik siehe Wrba, Dolznig \& Mannhalter (2007/2011). Für eine knappe Beschreibung der historischen Etablierung und Entwicklung der Genetik als Disziplin von den

7 Anzumerken ist hier wiederum, dass es sich beim vorliegenden Begriff - hier also dem der Pangenesis(theorie) - um kein neuartiges Gedankenkonstrukt handelt, sondern um eines, welches sich zumindest in rudimentärer Form bis zu Naturphilosophen der Antike zurückverfolgen lässt. 
Beiträgen durch Mendel im 19. Jahrhundert bis zur jüngeren Forschungsgeschichte einschließlich der Epigenetik sei Gayon (2016) genannt. Für eine umfassende Einführung in mathematische Modelle zur Biologie (bspw. zur Populationsdynamik) vergleiche Murray (1989/2008). Zuletzt sei nochmals auf die bereits genannten Einführungen zur Evolutionsbiologie verwiesen, denn die nachfolgenden Erläuterungen und Beschreibungen weisen zugunsten der Verständlichkeit und Knappheit einen hohen Abstraktionsgrad auf und können die Lektüre einschlägiger Einführungen nicht vollends ersetzen.

\subsection{Die wilde und ungesteuerte Natur der evolutionären Dynamik}

Zur Veranschaulichung der gemeinsamen Abstammung aller Lebensformen findet oftmals die bildhafte Beschreibung sowie die bildliche Darstellung eines „Stammbaum des Lebens“ Verwendung. Diese populäre Metapher scheint im ersten Moment zielführend, impliziert jedoch ein tiefgreifendes Missverständnis gegenüber der Art und Weise, in der sich die biologische Evolution vollzieht. ${ }^{8}$ In den mit einem solchen „Stammbaum“ verbundenen taxonomischen Darstellungen ist eine Klarheit und - trotz der gelegentlichen Trennung von Abstammungslinien - Linearität, ja geradezu Zielgerichtetheit, impliziert, welche die biologische Realität verfälschend abbildet. Evolution in vivo ist im Gegensatz hierzu vielmehr als ein äußerst buschiger Prozess aufzufassen, dessen langfristige Ergebnisse erst in einer retrospektiven Betrachtung, nachdem sich die Wogen dieses sehr unübersichtlichen eigentlichen Prozesses einmal gelegt haben, einem Stammbaum zu gleichen scheinen.

Um dies zu verdeutlichen, sei sich vorzustellen, wie ein jedes tierische Individuum - einschließlich einzelner Menschen - ein Agglomerat verschiedentlicher Eigenschaften, die innerhalb der jeweiligen Art, der das Individuum zugehörig ist, vorkommen, darstellt. ${ }^{9}$ In diesem Sinne ist ein jedes Individuum

8 Tatsächlich sind zwei missverständliche Auffassungen auf Basis dieser Darstellungsweise möglich. Die Baum-Metapher kann prinzipiell als ein Plädoyer zugunsten der Entwicklung von simplen oder „niedrigeren“ hin zu komplexeren oder „höheren“ Lebensformen, einschließlich des Menschen als „Krone der Schöpfung“, missverstanden werden - und wurde historisch oftmals auf diese Weise aufgefasst. Da eine solche Interpretation in heutiger Zeit jedoch gemeinhin nicht mehr vertreten wird, sei auf eine weitere Diskussion hierzu zu verzichten.

9 Diese phänotypische Variation begründet sich freilich in größtem Maße direkt auf der zwischen Individuen gegebenen genotypischen Variation. Als die populär bekanntesten Faktoren, welche diese Variation maßgeblich bestimmen, sind hier Mutation und Rekombination zu 
wahrlich einzigartig. Zugleich existiert jedoch auch umfangreich Übereinstimmung zwischen einzelnen Individuen innerhalb einer Art - weshalb es auch sinnvoll ist, die Gesamtpopulation dieser Individuen als eine gemeinsame Art $\mathrm{zu}$ beschreiben und auf diese Weise von anderen Arten abzugrenzen. Beide Feststellungen sind für sich genommen freilich nicht besonders überraschend. Nun ist jedoch darüber hinaus festzustellen, wie innerhalb dieser Gesamtpopulation einzelne Individuen nicht in gleichem Maße voneinander verschieden sind. Stattdessen besteht eine mittlere Beschreibungsebene zwischen dem Individuum und der Gesamtpopulation, welche als eine Summe insularer Eigenschaftshäufungen aufgefasst werden kann. Die Verteilung dieser Eigenschaftshäufungen ist teils durch regionale Verbreitung und ökologische Einflüsse zu erklären - örtlich abweichende Gegebenheiten führen sicherlich zur Ausbildung einer spezifisch regionalen Eigenschaftshäufung im Sinne jener mittleren Beschreibungsebene - , jedoch reichen in einer hinreichend großen Population schon allein statistische Prinzipien aus, um einen großen Anteil solcher bestehenden insularen Eigenschaftshäufungen zu erklären.

Die Bedeutsamkeit des eben Erläuterten liegt nun in der Feststellung, dass eine solche auf mehreren Ebenen stattfindende, höchst multidimensionale Dynamik ein hervorragender Nährboden für den evolutionären Prozess darstellt. Mittels der Ausbildung einer Vielzahl von Individuen mit konkreten Eigenschafts-Kombinationen nebst statistischen und regionalen Teilpopulationen mit konkreten Eigenschafts-Häufungen entsteht ein äußerst kraftvolles Potenzial zur Wandlung der Art oder von Teilpopulationen unter einem geeigneten selektiven Einfluss - sowie seltener auch ohne eine solche externe Einwirkung. Ganz allgemein sei zusammengefasst: Die Variation innerhalb einer Art steigt über Zeit und fächert prinzipiell in alle potenziellen Richtungen aus. Dies gilt für alle obengenannten Beschreibungsebenen, wodurch ein mehrschichtiges bzw. mehrdimensionales Mosaik entsteht.

Hierbei stellt sich sicherlich die Frage, wie in einem derart chaotischen und beständig ausdifferenzierenden System überhaupt distinkte Arten existieren können bzw. wie sich diese Arten überhaupt als solche herausbilden haben können. Die Beantwortung dieser Frage begründet sich einerseits in selektiven Einflüssen auf diese beständige, ungesteuerte Variation und andererseits in der Berücksichtigung von populationsdynamischen Prinzipien. Zunächst sei festzu-

nennen, ohne dass hierzu an gegebener Stelle Weiteres zu erläutern wäre, denn auch beim geneigten Laien ist oftmals einschlägig wenigstens ein rudimentäres, ausreichendes Bewusstsein gegeben - begründet durch informative Einflüsse aus der populären Kultur oder gegebenenfalls noch aus Schulzeiten verblieben. 
stellen, dass Arten eine hohe Stabilität aufweisen können, solange sie bereits gut an eine ökologische Nische innerhalb eines stabilen Ökosystems angepasst sind. Individuelle Variation und Eigenschaftshäufungen, welche von dieser stabilen Situation einer leistungsstarken Angepasstheit wegführen bzw. generell eine gewisse, hier notwendig vage formulierte, „Grenze der Andersheit“ überschreiten, stehen unter einem negativen Selektionsdruck - d. h. der auf der jeweiligen Art einwirkende selektive Einfluss begünstigt nicht einen Wandel, sondern stabilisiert die vorhandene Form, und zu sehr abweichende Variation geht innerhalb der weiterhin stattfindenden evolutionären Dynamik verloren. ${ }^{10}$ In anderen Worten: Solange eine ökologische Situation stabil ist und ein Organismus innerhalb dieser ökologischen Situation gut angepasst ist, so gilt in der Regel, dass der evolutionäre Prozess der Selektion diejenigen individuellen oder gehäuften Eigenschaften, welche an den äußersten Enden bzw. Rändern der Variation innerhalb einer Population liegen, durch negative Selektion beständig beschneidet - ähnlich einem Gärtner, der die Büsche seines Gartens regelmäßig in Form stutzt. Dennoch gilt, dass die Varianz innerhalb einer Art über Zeit zumindest prinzipiell zunimmt, sodass das einer Art inhärente Potenzial zur Wandlung hoch bleibt bzw. erhöht wird. Um zugunsten jenes letzten Punktes bei der Metapher des Gärtners zu bleiben: Auch während der Busch regelmäßig gestutzt wird, so wächst, verästelt und verdichtet er innerhalb der ihm verbliebenen Form.

Insbesondere dann, wenn sich eine gegebene ökologische Situation als instabil zeigt und wenn sich damit die Bedingungen der existierenden selektiven Einflüsse ändern, ${ }^{11}$ so wird zum einen das inhärente Wandlungspotenzial der Art aktiviert und zum anderen die ohnehin durchweg bestehende Tendenz bzw. der ohnehin beständig stattfindende Prozess, allseits in alle möglichen Richtung aufzufächern, begünstigt. Das Resultat ist ein äußerst buschiges natürli-

10 Eine Elimination aus dem Genpool in diesem Sinn kann freilich auf unterschiedliche Weise geschehen: Sollte ein Individuum einen besonders abweichenden Phänotyp entwickeln, so ist hinsichtlich dieses Individuums je nach Umfang der Abweichung mehr oder weniger stark erschwert, einen Sexualpartner zu finden. Auch externe Faktoren erschweren eine Fortpflanzung - beispielsweise im Fall eines Beutetiers unter der Voraussetzung, dass ein abweichender Phänotyp eine Verminderung der Angepasstheit aufgrund einer erhöhten Bejagung bedeutet. Zuletzt kann eine kleinere Teilpopulation freilich auch schlicht auf statistischer Basis im größeren Genpool aufgehen, sodass die Nachkommen spezifische Eigenschaftshäufungen der vorherigen Generation(en) wieder verlieren.

11 Es handelt sich hierbei um ein sehr prototypisches Szenario. Grundsätzlich kann auch ein Gendrift allein - als ein bedeutender Evolutionsfaktor - die evolutionäre Entwicklung einer Art nennenswert beeinflussen. 
ches Experimentieren, in dem die Gesamtpopulation ihr ohnehin komplexes und mehrschichtiges Mosaik nochmals erweitert. Fachsprachlich handelt es sich bei diesem Vorgang um eine adaptive Radiation.

Diese gegebenenfalls äußerst dynamische Situation endet nicht zwingend in der Entwicklung unterschiedlicher Arten. So ist möglich, dass sich die Gesamtpopulationen nicht in hinreichend getrennte Teilpopulationen aufspaltet, wodurch die Art in ihrer Gesamtheit eine genotypische und phänotypische Verschiebung durchläuft, anstatt sich zu trennen. Jedoch ist ebenso möglich, dass sich in diesem Ereignis zwei oder mehrere distinkte genotypische, und damit zugleich phänotypische, Traditionen herausbilden - die Geburt von getrennten Linien, welche nach einer entsprechend langen und umfangreichen Entwicklung potenziell in jeweils eigenen Arten münden. Dass sich in einer solchen Situation in der Regel nur eine geringe Anzahl neuer Arten herausbildet und nicht - gemäß der beschriebenen Situation einer buschigen und wilden Radiation in praktisch alle möglichen Richtungen - eine Vielzahl neuer Arten, ist darin zu begründen, dass die entstehenden Varianten typischerweise in Konkurrenz zueinander treten, ${ }^{12}$ womit ein Großteil der entstandenen Varianten innerhalb eines mehr oder weniger kurzen Zeitraums wieder unterbunden wird. Retrospektiv verbleibt auf diese Weise eine ästhetisch ansprechende Astgabelung des Stammbaums des Lebens - während der evolutionäre Prozess in vivo dagegen einen äußerst komplexen, schnelllebigen, undurchsichtigen und distinkt nicht-linearen Vorgang darstellt.

Es sei damit nochmals knapp zusammengefasst: Unter der Voraussetzung, dass eine gegebene Art bereits sehr gut an ihre Position in einem stabilen Ökosystem angepasst ist, so erhält sich eine gewisse Stabilität der Gesamtpopulation dadurch, dass es die Randbereiche jener beständig auftretenden Variation sind, welche durch den bestehenden Selektionsdruck zugunsten der bereits angepassten Form abgeschnitten bzw. ausgesondert werden. Jedoch in der Situation, in welcher sich eine bestimmte Form - oder mehrere Formen - des Eigenschaften-Mosaiks als vorteilhaft erweist bzw. erweisen, führt die entsprechende adaptive Radiation unter fördernden Umständen $\mathrm{zu}$ einer Trennung in zwei oder mehrere Linien, welche potenziell in eigenen Arten münden. Jenseits dieser prototypischen Dynamik sind innerhalb der evolutionsbiologischen Realität freilich höchst diffizile Szenarien möglich, welche von weiteren Evolutionsfaktoren abhängig sind.

12 Dies sowohl hinsichtlich der ökologisch gegebenen Ressourcen als auch in genotypischer Hinsicht - Letzteres zumindest, solange es sich um eine zur Fortpflanzung befähigte Gesamtpopulation handelt. 


\subsection{Eldredge \& Goulds Punktualismus und Implikationen für das Bisherige}

Mit diesen Beschreibungen zur zentralen Dynamik des evolutionären Vorgangs durchaus verbunden ist eine Diskussion zum Konzept des Punktualismus (punctuated equilibrium), der in den 1970ern populär durch Gould - im Anschluss an eine primär durch Eldredge geprägte Veröffentlichung (Eldredge \& Gould 1972; vgl. zu Eldredges Rolle Gould 1991) - in die Diskussion zur evolutionären Änderungsrate eingeführt wurde und jahrzehntelang ein zentraler Diskussionspunkt der jüngeren Forschungsgeschichte war. ${ }^{13}$ Aufgrund der Prominenz dieses Konzepts soll es nachfolgend zweckmäßig knapp beschrieben und innerhalb der bisherigen Erläuterungen verortet werden. Zugleich können damit etwaige Missverständnisse, welche innerhalb der populären Literatur und innerhalb des durch Laien geprägten kulturellen Gedächtnisses gegenüber jener Position weitläufig bestehen, ausgeräumt werden.

Hintergrund zum Konzept des Punktualismus ist die Feststellung, dass Ergebnisse der evolutionsbiologischen Forschung - darunter besonders Fossilfunde - suggerieren, dass taxonomische Gruppen hinsichtlich ihres Phänotyps lange Zeit vergleichsweise stabil bleiben können, um dann in geologisch kurzen Zeiträumen umfangreiche morphologische Umgestaltung zu durchlaufen. Beispiele zu Taxa, welche über geologisch lange Zeiträume kaum phänotypische Veränderungen zeigen, sind überraschend leicht zu identifizieren: Beispielhaft seien Krokodile und Haie genannt, welche beide hinsichtlich ihrem durch Fossilfunde dokumentierten Phänotyp keine groben Veränderungen während der letzten 200 Millionen Jahre zeigten.

Diese Feststellung erscheint im ersten Moment nur schwer vereinbar sowohl mit einem sukzessive verlaufenden Gradualismus als auch mit der Kenntnis darum, dass der evolutionäre Prozess im oben beschriebenen Sinn bestän-

13 Wieder einmal ist sowohl der Begriff als auch die hintergründige Idee nicht „spontan“ innerhalb der jüngeren Forschung entstanden. Der Begriff des Punktualismus ist - wenn auch in Form einer abweichenden Interpretation bzw. Verwendung - wesentlich älter als hier angegeben. Der aus heutiger Sicht veraltete Begriff eines Punktualismus verneint die graduelle, evolutionäre Veränderung von Organismen über Zeit und nimmt eine sprunghafte Änderung von einer Art zur nächsten an. Der Punktualismus in der hier verwendeten Form bezieht sich auf die variable und teils erstaunlich schnelle Änderungsrate, mit der eine ansonsten prinzipiell gradualistische Veränderung fortschreiten kann - eine Interpretation, die im Kern nicht vollends neu ist, sondern auf bestehendem Gedankengut aufbaut bzw. sich dabei teilweise in einen bewussten Kontrast setzt (vgl. bspw. Mayr 1954 als eine in diesem Sinne wegbereitende Veröffentlichung). 
dig und äußerst „buschig“ stattfindet. Dennoch ist eine angemessene Antwort aus dem bereits Beschriebenen durchaus ableitbar: Ist ein Organismus innerhalb eines stabilen Ökosystems und hinsichtlich seiner ökologischen Nische angepasst, so gilt gemeinhin, dass Variation, welche von dieser gut angepassten, gleichgewichtigen Situation wegführt, durch negative Selektion bereinigt bzw. ausgesondert wird. Auf diese Weise kann ein Taxon über lange Zeiträume morphologisch äußerst einheitlich erscheinen, ohne dass die prinzipielle Realität des evolutionären Prozesses auf dieser Basis zu verneinen ist. Ebenso ist die vergleichsweise sprunghafte Radiation teils damit zu erklären, dass u.a. Änderungen im Ökosystem dazu führen können, dass eine adaptive Radiation evolutionäre Wege schafft, die zuvor lange Zeit verwehrt geblieben waren - womit das inhärent Wandlungspotenzial der Art „entfesselt“ wird. ${ }^{14}$

In ähnlicher Weise wie soeben beschrieben erkennen auch die Diskussionsgegner des modernen Punktualismus im Sinne des punctuated equilibrium das vorgeschlagene Wandlungsmuster von Arten - also die höchst variable Rate phänotypischer Evolution - an. Dawkins' variable speedism integriert die genannten zentralen Überlegungen des Punktualismus in seine eigene Position (vgl. zu Dawkins' Auseinandersetzung mit Eldredges und Goulds Punktualismus Dawkins 1986/2006: 326-360 sowie darin konkret zum variable speedism insbesondere ab 350). Dies bedeutet einerseits, dass die Meinungsverschiedenheiten jener Biologen erst jenseits dieser die evolutionäre Dynamik bestimmenden Prozesse beginnen, und andererseits, dass für die vorliegende Skizze ebenjener zentralen evolutionären Dynamik keine weiterführende Klärung vonnöten ist, sodass $\mathrm{zu}$ anderen Punkten vorangeschritten werden kann - wobei mit den nachfolgenden Worten zunächst noch ein zwischenzeitliches Fazit gezogen werden soll.

Das Bisherige stellt den Versuch dar, mit nur wenig fachsprachlichem Jargon und nur der notwendigsten Fachterminologie sowohl eine historische Platzierung der modernen Evolutionstheorie vorzulegen als auch die innerste Dynamik biologischer Evolution anschaulich zu skizzieren. Dies soll zugunsten späterer Diskussionen einen hilfreichen Verständniskontext schaffen. Das vorliegende Kapitel stellt dagegen nicht den Versuch dar, simple und laienhafte

14 Ein weiterer und hier nicht zu unterschlagender Faktor, welcher das Erscheinungsbild von Stillstand und Sprunghaftigkeit im evolutionären Prozess zusätzlich verstärkt und damit teilweise zu erklären vermag, betrifft - wenigstens innerhalb der Paläontologie - schlicht die prinzipiell nicht erschöpfende Fundlage. Da fossile Funde innerhalb einer evolutionären Linie oftmals mehrere Millionen Jahre auseinanderliegen, ist zwischen solchen Funden oftmals ein nennenswerter „Sprung“ feststellbar, ohne dass die evolutionsbiologische Dynamik hinterfragt werden müsste. 
Vorurteile gegenüber der Evolutionstheorie zu erhellen. ${ }^{15}$ In diesem Sinne ist das Gegebene als weitgehend ausreichend und damit abgeschlossen festzustellen. Lediglich eine letzte Klärung sei im Sinne des Programms des vorliegenden Kapitels noch als wertvoll anzusehen und soll daher im Nachfolgenden knapp diskutiert werden, um spätere Abschnitte der vorliegenden Arbeit in ähnlicher Weise $\mathrm{zu}$ fundieren wie mit dem bisher Vorgebrachten geschehen: Die trotz der buschigen und unübersichtlichen evolutionären Dynamik dennoch kostenbedachte und konservative Natur biologischer Evolution.

\subsection{Evolution als konservativer „Bastler“}

Eine wertvolle allgemeinsprachliche Umschreibung dieses dem Artwandel zugrundeliegenden Prinzips ist das durch Jacob (1977) ins populäre Bewusstsein gebrachte Motiv der Evolution als ein „Bastler“. In diesem Sinne ist zunächst nochmals explizit zu betonen, dass der evolutionäre Weg hinsichtlich konkreter körperlicher Ausprägungen prinzipiell graduell geschieht und mit jedem Schritt gezwungenermaßen sukzessive auf dem jeweils bisherigen Stadium aufbaut. Daraus ist einerseits zu folgern, dass eine jede ausgebildete Körperform in ihrer heutigen Konstitution und hinsichtlich aller ihrer Teile einen langen, von Zwischenstufen reichen Weg beschritten hat. Andererseits - und damit soll im gegebenen Kontext der Kern des Diskurses gegeben sein - korreliert diese durch Akkumulation sukzessive herausgebildete, konkrete Form stark mit einer im Verlauf dieser Herausbildung zunehmenden Tendenz zur Konservation gegenüber dem bereits Erreichten, solange ein entsprechender Selektionsdruck gegenüber diesem Erreichten fortbesteht.

Zur Verdeutlichung des Gemeinten sei ein konkretes Beispiel genannt: Praktisch alle höheren terrestrischen Wirbeltiere besitzen einen neuronalen Komplex, der die Kontraktion der Hand bzw. Tatze inklusive Finger respektive Krallen steuert. Während hier innerhalb der Säugetiere eine Homologie - also die Übereinstimmung der Körperstruktur aufgrund eines gemeinsamen evolutionären Ursprungs anstatt einer unabhängigen analogen Entwicklung - ge-

15 Aus diesem Grund sei auf die Aufklärung von Aussagen wie „Die Evolutionstheorie ist nur eine Theorie.“ und „Evolution ist das Überleben des Stärkeren.“ sowie auf die Beantwortung von Fragen wie „Welchen Nutzen hat ein halbes Auge?“ und „Wenn der Mensch vom Affen abstammt, warum gibt es dann noch Affen?“ gänzlich zu verzichten. Die vorliegende Arbeit sieht ihren Diskurs jenseits eines derart naiven Standards und die populäre Literatur bietet im Rahmen der einfachsten Recherche ausreichend Aufklärung jener höchst fraglichen Feststellung bzw. Fragen. 
meinhin nicht bezweifelt wird, so wird die wahre evolutionäre Tiefe dieser konkreten Struktur durchaus diskutiert und umfasst die höheren terrestrischen Lebewesen möglicherweise weitläufig (vgl. Iwaniuk \& Whishaw 2000 sowie Fontanarrosa \& Abdala 2016). Unabhängig vom tatsächlichen Alter dieser in jedem Fall durch Homologie weit verbreiteten körperlichen Funktionalität ist festzustellen, dass dieselbe von überlebensnotwendiger Wichtigkeit für eine Vielzahl von Tiergruppen ist - immerhin fundiert diese Struktur eine große Zahl bedeutender Verhaltensweisen wie das Packen und Festhalten von Beutetieren, ein leistungsfähiges Klettern und nicht zuletzt die Handhabung von Gegenständen. Trotz der immensen Bedeutung dieses basalen neuronalen Mechanismus für eine Vielzahl verschiedener Arten in Kombination mit ihren jeweiligen biomechanischen Gegebenheiten bzw. Anforderungen ist diese primitive neurologische Ausprägung in vielen konkreten Fällen nicht ausreichend. Innerhalb der Primaten entwickelten sich so beispielsweise zusätzliche Systeme, welche die biomechanische Komplexität des Greifens stark erhöhten (vgl. Schieber 1995 für eine einschlägige Beschreibung). Insbesondere der menschliche Präzisionsgriff erfordert selbst im Vergleich mit anderen Affen eine außerordentlich freie Beweglichkeit der Hand und spezifischer Finger - im Gegensatz zu verschiedensten Arten, in denen entweder eine allgemeine Kontraktion des Greiforgans oder eine durchaus komplexe, aber dennoch nicht im menschlichen Maße ausgebildete, Ausrichtung der Fingerposition ausreicht. ${ }^{16}$

Die Herangehensweise eines Ingenieurs in der Aufgabe, den Schritt von einem simpleren System zu einem komplexeren anzugehen, wäre klar: Das alte System würde abgeschafft und durch ein neues ersetzt. Im Sinne der bisherigen Erläuterungen ist eine solche Vorgehensweise innerhalb der biologischen Evolution allerdings undenkbar. Intuitiv eingängiger bzw. mit biologischer Evolution scheinbar vereinbarer wäre es, das alte System sukzessive zu erweitern. Hier ergibt sich jedoch diejenige Problematik, welche die vorliegende Diskussion motiviert: Ein jede Abweichung von einem bereits durch Adaptation etablierten Mechanismus trübt - wenigstens vorübergehend - die Leistungsfähigkeit dieses Mechanismus im Kontext des nur graduellen Fortschreitens evolutionärer Veränderungen. Im Fall des Beschriebenen neuronalen Komplexes ist beispielsweise vorstellbar, dass unter anderem durch Interferenzen innerhalb des nicht differenzierenden Nervenstrangs Einschränkungen der Funktionalität entstehen - womit das prinzipielle Weiterbestehen dieses Systems durch nega-

\footnotetext{
16 Prinzipiell ist die freie Beweglichkeit einer oder mehrerer Finger freilich kein Alleinstellungsmerkmal des Menschen, sondern ist in unterschiedlich großem Umfang in anderen Arten gegeben. Die gemachten Feststellungen werden hiervon dennoch nicht eingeschränkt.
} 
tive Selektion gestützt wird. Um also die bestehende Funktionalität nicht einzuschränken und trotzdem dem Selektionsdruck hin zum leistungsfähigeren Greifen nachgeben zu können, entsteht ein ergänzendes und hierarchisch übergeordnetes System, welches in der Lage ist, die „gewünschte“ neuartige Funktionalität zu fundieren, ohne auf dem Weg dorthin die bisherige Funktionalität einzuschränken. Über mehrere solcher Erweiterungen entsteht im weiteren evolutionären Verlauf ein mehrschichtiges Konglomerat verschiedener Signaltypen, welches auf hochkomplexe und aus Sicht des Ingenieurs unnötig komplizierte Weise die Hand- bzw. Fingerbewegung zu steuern vermag. Auch dem leistungsfähigen, fingerspezifischen Präzisionsgriff des Menschen ist es nur auf intuitiv wenig naheliegende Weise möglich, das gezielte Steuern einzelner Finger zu vollbringen - so beispielsweise primär nicht, indem ausdifferenzierte Signale gezielt an einzelne Finger bzw. deren Muskulatur gesendet werden, sondern indem das Signal bzw. die Signale ursprünglicherer Systeme hinsichtlich einzelner Finger inhibiert bzw. abgeändert werden. ${ }^{17}$

Auch das Gehirn stellt ein hervorragendes Beispiel solcher stufenweiser Flick- bzw. Bastelarbeit dar: Stammhirn bzw. Hirnstamm, Zwischenhirn und Kleinhirn erfüllen im Groben noch immer diejenigen Aufgaben, für die sie im evolutionären Verlauf aufgekommen sind, und wurden innerhalb eines wiederkehrend erweiterten Gesamtkomplexes integriert - anstatt fundamental abgeändert zu werden. Nur auf diese Weise kann eine beständig leistungsfähige Funktionalität gewährt werden, während zugleich ein evolutionäres Fortschreiten nicht gehindert wird.

Dies sei damit die allgemeine, spätere Abschnitte fundierende Feststellung zum vorliegend Diskutierten: Evolutionärer Wandel erfolgt oftmals höchst konservativ. Etablierte Körperformen oder Systeme wandeln sich nicht vollständig, sondern gleichen einem innerhalb einer Einbahnstraße zurückgelegten Weg, der - gemäß ihrer Natur als metaphorische Einbahnstraße - nicht mehr bzw. nur äußerst erschwert umkehrbar ist. ${ }^{18}$ Dies stellt jedoch zugleich den evolutio-

17 Vgl. Castiello (2005) für einen Überblick zur Beweglichkeit der menschlichen Finger und zum Greifen der Hände. Vgl. Schieber et al. (2010) für konkrete Diskussionen zu den neuronalen Strukturen des menschlichen präzisen Greifens einschließlich der inhibierenden neuronalen Mechanismen im soeben beschriebenen Sinn im Kontext pathologischer Fälle. Vgl. zusätzlich Mollazadeh (2014) für Untersuchungen zu Synergieeffekten innerhalb der neuroanatomischen bzw. auch muskulären Repräsentation von Hand- und Fingerbewegungen innerhalb der beschriebenen, äußerst komplexen biologischen Realität des Greiforgans beim Menschen sowie bei nicht-menschlichen Affen.

18 Die wenigstens prinzipielle Unumkehrbarkeit wird durch den Fall von funktionslos gewordenen und damit sukzessive rückgebildeten Organen - beispielhaft seien die Hinterbeine samt 
nären Wandel, wie anhand der beschriebenen Beispiele festhalten werden kann, nicht in Frage. Die evolutionäre Dynamik erlaubt großflächigen und weitläufigen Wandel trotz der diskutierten Einschränkungen: Wale sind relativ nahe Verwandte von Paarhufern wie Flusspferden, Kamelen, Wiederkäuern und Schweinen (Thewissen et al. 2007) und Menschen sind ein Taxon innerhalb der Affen, das mittels Raketentechnologie den Mond betreten hat.

In diesem Sinne können auch außerordentlich dynamische adaptive Radiationen wie die kambrische Artenexplosion in einen adäquaten Kontext gesetzt werden: Sobald eine Radiation prinzipielle Körperbaupläne oder auch kleinere Innovationen in den Weg geleitet hat, so sind die daraus abgeleiteten Arten zur Innovation gezwungen, denn eine Möglichkeit zur Revolution ist nicht mehr gegeben. Auf diese Weise kann das moderne Huhn eine evolutionäre Nähe zu etwas morphologisch derart Abweichendem wie Tyrannosaurus rex aufweisen, ohne dabei in der Lage zu sein, den evolutionären Ballast vollständig abzulegen.

Becken von Individuen innerhalb der Linie hin zu modernen Walen genannt - nicht in Frage gestellt, denn zur Diskussion stehen nicht funktionslos gewordene, sondern durchgängig funktionstragende Organe. 